\title{
Development of Nanosecond Laser Module Built in the Robotic Muttifunctional Surgical Complex for Minimally Invasive Therapy of Maxillofacial Area Pathology and Estimation of its Effects on Blood Plasma
}

D01. 10, $17691 / \mathrm{stm} 2016.8 .4 .04$

Received February 22, 2016

A.A. Chunikhin, MD, PhD, Associate Professor, Department of Oral Surgery';

M.X. Sahakyan, MD, PhD, Associate Professor, Department of Orthopedic Stomatology and Orthodontics; Chief Doctor of the Dental Implantology Center';

S.I. Gazhva, MD, DSc, Professor, Head of the Department of Dentistry, Faculty of Doctors' Advanced Training2; E.A. Bazikyan, MD, DSc, Professor, Head of the Department of Oral Surgery ${ }^{1}$

${ }^{1}$ Moscow State University of Medicine and Dentistry named after A.I. Evdokimov, 20/1 Delegatskaya St., Moscow, 127473, Russian Federation;

${ }^{2}$ Nizhny Novgorod State Medical Academy, 10/1 Minin and Pozharsky Square, Nizhny Novgorod, 603005, Russian Federation

The aim of the investigation was to assess the efficiency of singlet oxygen excitation in blood plasma in vitro using infrared laser radiation with $1,260 \pm 4 \mathrm{~nm}$ wavelength generated by a pulsed laser module, which was designed to be built in the robotic surgical device for minimally invasive maxillofacial surgery.

Materials and Methods. The model of the developed nanosecond laser (1,260 $\pm 4 \mathrm{~nm}$ wavelength) generating pulse signal with $400 \mathrm{~ns}$ pulse duration, $500 \mathrm{kHz}$ pulse repetition frequency, and $500 \mu \mathrm{m}$ optic fiber diameter was used in the investigation. Test tubes with heparin sodium having various centrifuging time served for obtaining blood plasma. Three blood plasma specimens obtained in different centrifuging modes were used in the study. Photochemical measurements were performed in blood plasma diluted with a neutral (pH 7.6) phosphate buffer. The reaction of releasing singlet oxygen was controlled by discoloration with the help of a spectrophotometer under laser radiation of the main band of the trap.

Results. The reduction of optical density at $414 \mathrm{~nm}$ spectrophotometer wavelength was significantly noted in all three specimens, the effect amounted to $0.04 \pm 0.01$ over 30 min of radiation. When buffer diluted blood plasma was exposed to the tested infrared laser during $1.5 \mathrm{~h}$, the significant effect of the trap discoloration was observed in all specimens. Laser radiation in the nanosecond pulse mode provided discoloration 10 times greater than that of the laser working in the continuous mode.

Conclusion. The experiments performed showed that the application of the original nanosecond pulsed laser module provided effective generation of singlet oxygen. Small dimensions and unique characteristics allow the module to be built in the robotic surgical complex.

Key words: robot-assisted surgery; nanosecond pulsed lasers; photodynamic effect; singlet oxygen.

Modern medicine is inconceivable without application of laser technologies. Laser radiation possesses unique properties with respect to biological tissues. A parallel beam of the light flux, generated by laser radiation, makes it possible to be focused on the object in the form of a spot with a minimal diameter and various parameters of exposure - wavelength, duration, intensity - allowing different reactions to be excited in the biological tissues [1].

The last decade showed an active development of robotic technologies in medicine. Design and implementation of da Vinci robot enabled surgeons to perform delicate surgical interventions in urology, gynecology, abdominal surgery [2]. Robotic laserbased surgical systems found their application in ophthalmology.

The results achieved convincingly show such advantages of robotic surgery as minimal invasiveness, high precision, and rapid recovery.

A very promising is the development of a robotic complex for treating complicated pathology of maxillofacial area by integration of a laser module to the robot-surgeon manipulators delivering light fluxes of laser radiation to the deep structures of the head and neck. Numerous tiny anatomic structures in the maxillofacial area - muscles, vessels and nerves, air-bearing

For contacts: Andrey A. Chunikhin, e-mail: docca74@yandex.ru 
sinuses - cause difficulties in surgical interventions and do not allow surgeons to make accurate manipulations [3]. Multiplicity of pathologies in the maxillofacial area, the necessity of using various methods of therapy, replacement and reconstructive surgery of the tissue and organs of the head and neck require mathematical calculation of conditions for procedure performance and application of artificial intelligence [4].

One of the stages of designing such multifunctional complex is the development and creation of a laser module with unique characteristics of laser radiation and possibility of combined exposure to low-intensity and high-intensity radiation for achieving instantaneously therapeutic and surgical effect in the tissues.

The effect of laser radiation on oxygen is one of the important factors determining biochemical reactions in the tissues. Photochemical reactions, excited in the cells under the action of laser radiation, trigger various molecular mechanisms in the tissues connected, among other things, with the transition of molecular oxygen dissolved in the tissues to a singlet state. Method of photodynamic therapy, which represents an interaction of three components (photosensitizer, laser radiation and oxygen) is based on photochemical reactions. Photosensitizer oxygen, accumulated in the tissues under the action of laser radiation, is converted to the excited triplet state, whose energy, in its turn, is transferred to the intracellular oxygen molecules with formation of molecular singlet oxygen according to the following scheme:

$$
\begin{gathered}
\text { Sens }+ \text { hu } \rightarrow{ }^{1} \text { Sens } \\
{ }^{1} \text { Sens } \\
{ }^{3} \text { Sens } \\
{ }^{3} \text { Sens } \\
{ }^{*} \mathrm{O}_{2}+{ }^{3} \mathrm{O}_{2} \rightarrow{ }^{1} \mathrm{O}_{2}+\text { Sens; } \\
{ }^{1} \text { Substrate } \rightarrow \text { Oxidation, }
\end{gathered}
$$

where singlet and triplet excited states of photosensitizer are represented as ${ }^{1}$ Sens* and ${ }^{3}$ Sens ${ }^{*}$, and singlet oxygen as ${ }^{1} \mathrm{O}_{2}$.

Singlet oxygen is a strong oxidizer of aromatic amino acids, and when reacting with cellular elements, it destroys membrane structures, initiating reaction of lipid peroxidation in a cell or pericellular space. Destruction of biological substrates results also in the generation of free radicals possessing a secondary damaging effect on the cells. There are a great number of compounds capable to work as photosensitizers, which can absorb radiation of all visible spectrum region, but a reaction rate increases many times when radiated by the light with a wavelength corresponding to the peak of absorption a narrow range specific for each individual compound. The possibility to conduct photodynamic reactions in the tissues without sensitizers by means of direct generation of singlet oxygen according to the following scheme:

$$
\begin{gathered}
{ }^{3} \mathrm{O}_{2}+\mathrm{hv} \rightarrow{ }^{1} \mathrm{O}_{2} ; \\
{ }^{1} \mathrm{O}_{2}+\text { Substrate } \rightarrow \text { Oxidation }
\end{gathered}
$$

is likely to be most perspective for using them in the medical practice. Such reactions occur when tissues are exposed to laser radiation with a wavelength approaching to the maximal absorption of oxygen in the tissues: $1,265 \pm 5 \mathrm{~nm}$ [5]. Application of ultrashort laser pulses (femtosecond, picoseconds, nanosecond) allows a light flux to penetrate deeper into the tissues without heating them. Due to a short lifetime and a high power of laser pulses during the period of electron transition, summation of the energy of two or even three laser photons takes place in the oxygen molecule, leading to filling the tissues with oxygen in its singlet state [6].

Laser emitters capable of generating ultrashort waves with a definite frequency have a high price and big dimensions [7]. A research team of the Moscow State University of Medicine and Dentistry named after A.I. Evdokimov has developed a model of diode laser generator with unique parameters. The generator is made as a compact module on the basis of semiconductor chips, capable of generating high power nanosecond pulses with 400 ns pulse duration and $500 \mathrm{kHz}$ pulse repetition frequency. Such characteristics provide deeper penetration of laser photons into the tissues and summation of their energy for increasing photodynamic effects without exogenous photosensitizers (application for invention No.2016129331 of 19.07.2016).

The given emitter may be applied as an independent laser device to be used in every-day practice of a dentist for various surgical interventions in the maxillofacial region, as well as in treating odontogenic inflammatory diseases and pathology of oral mucosa. Besides, small dimensions and flexible thin light fibers allow the application of this module in a robotic medical laser unit for minimally invasive maxillofacial surgery. To implement the new device into clinical practice and to ascertain its unique characteristics laboratory testing is required to assess different factors of laser impact on biological tissues including blood elements. One of the important aspects is to study photochemical effects in blood plasma exposed to laser radiation with definite parameters.

The aim of the study was to assess the efficiency of singlet oxygen excitation in blood plasma in vitro using infrared laser radiation with $1,260 \pm 4 \mathrm{~nm}$ wavelength generated by a pulsed laser unit which was designed to be built in the robotic surgical device for minimally invasive maxillofacial surgery.

Materials and Methods. A model of the original nanosecond laser with a sensor control display on semiconductor chips, having the basic infrared (IR) emitter with a wavelength corresponding approximately to the maximum of oxygen absorption (1,256-1,264 nm), generating pulse signal with 400 ns pulse duration and $500 \mathrm{kHz}$ pulse repetition frequency. Radiation was focused in the optical fiber having $500 \mu \mathrm{m}$ diameter.

The laser generator was designed in such a way as to be able to mount up to three additional emitters with various wavelengths to be used in one light fiber. This scheme is an advantage over the existing analogs, as an application of several wavelengths of multidirectional action in one fiber will enable manipulations 
simultaneously with different effects on tissues, promoting better therapeutic effect.

A test tube with heparin sodium and olefinic gel was used to isolate plasma from blood. Heparin is an acid mucopolysaccharide with a molecular weight of 3,000 to $40,000 \mathrm{Da}$. It is a natural anticoagulant activating creation of complex compounds between antithrombin III and such factors of blood clotting as thrombin, factors XIla, Xla, Xa, IXa and VIla.

In such a complex the clotting factors are irreversibly inactivated. Heparin sodium salt in the proportion of 15$20 \mathrm{ME} / 1 \mathrm{ml}$ to the blood collected into the tube ensures full inactivation of blood clotting factors and does not distort fluid parameters. Olefinic gel is a thixotropic polymer, which is heavier than plasma, but lighter than formed elements of blood, therefore after centrifuging it occupies an intermediate position in the form of a thin strip and serves as a separating barrier.

Photochemical measurements were carried out in blood plasma diluted by a neutral $(\mathrm{pH}$ 7.6) phosphate buffer (1,3-diphenylisobensofuran, DPIBF). Three specimens of blood plasma, obtained by different modes of centrifuging, were used for the experiments: specimen $1-10 \mathrm{~min}$ at $3,600 \mathrm{rpm}$ and $20 \mathrm{~min}$ at $6,000 \mathrm{rpm}$; specimen $2-10 \mathrm{~min}$ at $4,500 \mathrm{rpm}$ and $15 \mathrm{~min}$ at $3,600 \mathrm{rpm}$; specimen $3-15 \mathrm{~min}$ at $6,000 \mathrm{rpm}$. To provide a reliable measurement of optical density in $380-500 \mathrm{~nm}$ wavelength range of the spectrometer, plasma required twofold dilution by the buffer in each case, as it contained a noticeable number of hemoglobin.

Square quartz fluorescence cuvettes with $1 \mathrm{~cm}$ optical path length were used in the work. The volume of specimens in the cuvettes was always equal to $0.75 \mathrm{ml}$, and DPIBF concentration was selected in such a way that optical density in the maximum of absorption was within the range of 0.9-1.1. The fiber head was placed closely to the cuvette containing the trap solution. Duration of specimen radiation session was chosen so that the effect of DPIBF discoloration was reliably measurable, but not too strong. The optical density of the solutions (at $414 \mathrm{~nm}$ wavelength) was measured before and after the exposure using SF-56 spectrophotometer (Solar, Russia).

Reaction of singlet oxygen release was controlled by means of spectrophotometer by discoloration under laser radiation of the main band of trap absorption at $414 \mathrm{~nm}$. To determine accurately the responsibility of singlet oxygen for the decrease of the substrate, special chemical agents - singlet oxygen quenchers - were added to the solution. Besides, the solutions were made free of the dissolved oxygen by bubbling them with nitrogen.

In order to assess the efficiency of singlet oxygen generation using the developed module, the acquired data were compared with those obtained from 1,270 nm laser generator working in the continuous radiation mode.

Results and Discussion. All specimens of DPIBF solutions in the diluted blood plasma exposed to radiation by the tested IR laser showed a significant effect of trap discoloration during $1.5 \mathrm{~h}$ (Figures 1-3).

Reduction of optical density at $414 \mathrm{~nm}$ was significantly noted in all three blood plasma specimens, the effect amounted to $0.04 \pm 0.01$ over $30 \mathrm{~min}$ of radiation. High centrifuging speeds influenced the increased blood cell destruction (erythrocytes, leukocytes), causing a high content of antioxidant substances in the specimens obtained. Despite the availability of a high level of antioxidants responsible for intensive quenching of the singlet oxygen, a reliable decrease of the chemical trap density was registered.

Comparison of the efficiency of generating singlet oxygen by the developed nanosecond ultrashort pulsed laser module and a continuous mode laser emitter generating a wavelength approaching to the maximum of oxygen absorption $(\approx 1,270 \mathrm{~nm})$ gave the following results (See the Table).

The values of relative rates of trap discoloration in the blood plasma under the action of two IR lasers correspond

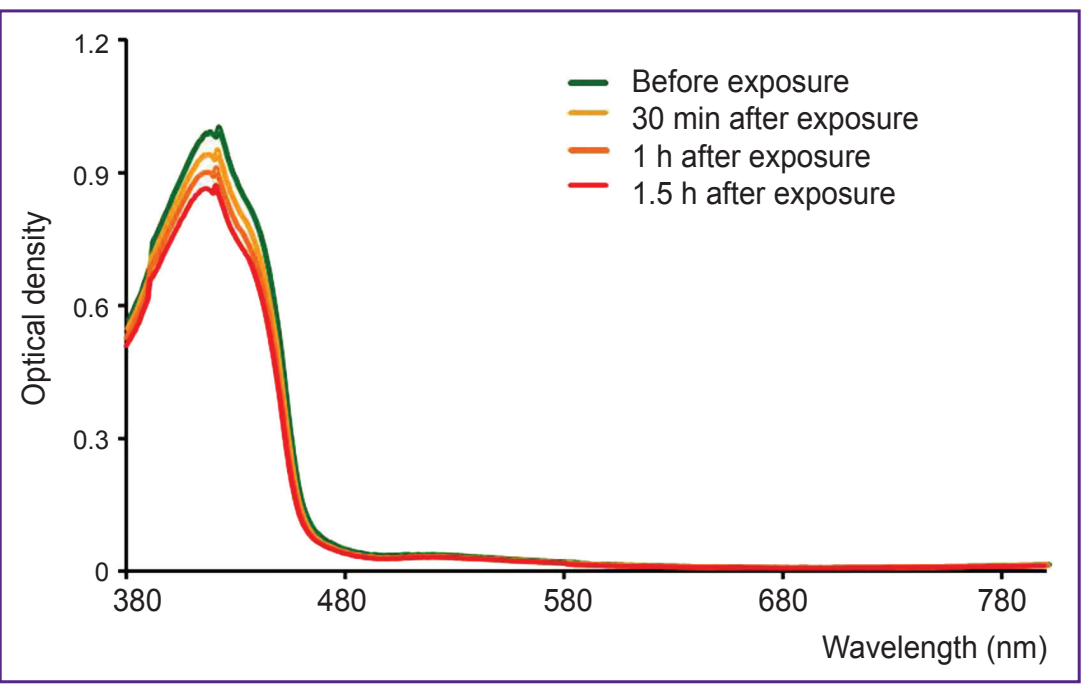

Figure 1. A series of absorption spectra of the chemical trap in the diluted blood plasma (specimen 1) exposed to the tested laser radiation 
Figure 2. A series of absorption spectra of the chemical trap in the diluted blood plasma (specimen 2) exposed to the tested laser radiation
Figure 3. A series of absorption spectra of the chemical trap in the diluted blood plasma (specimen 3) exposed to the tested laser radiation
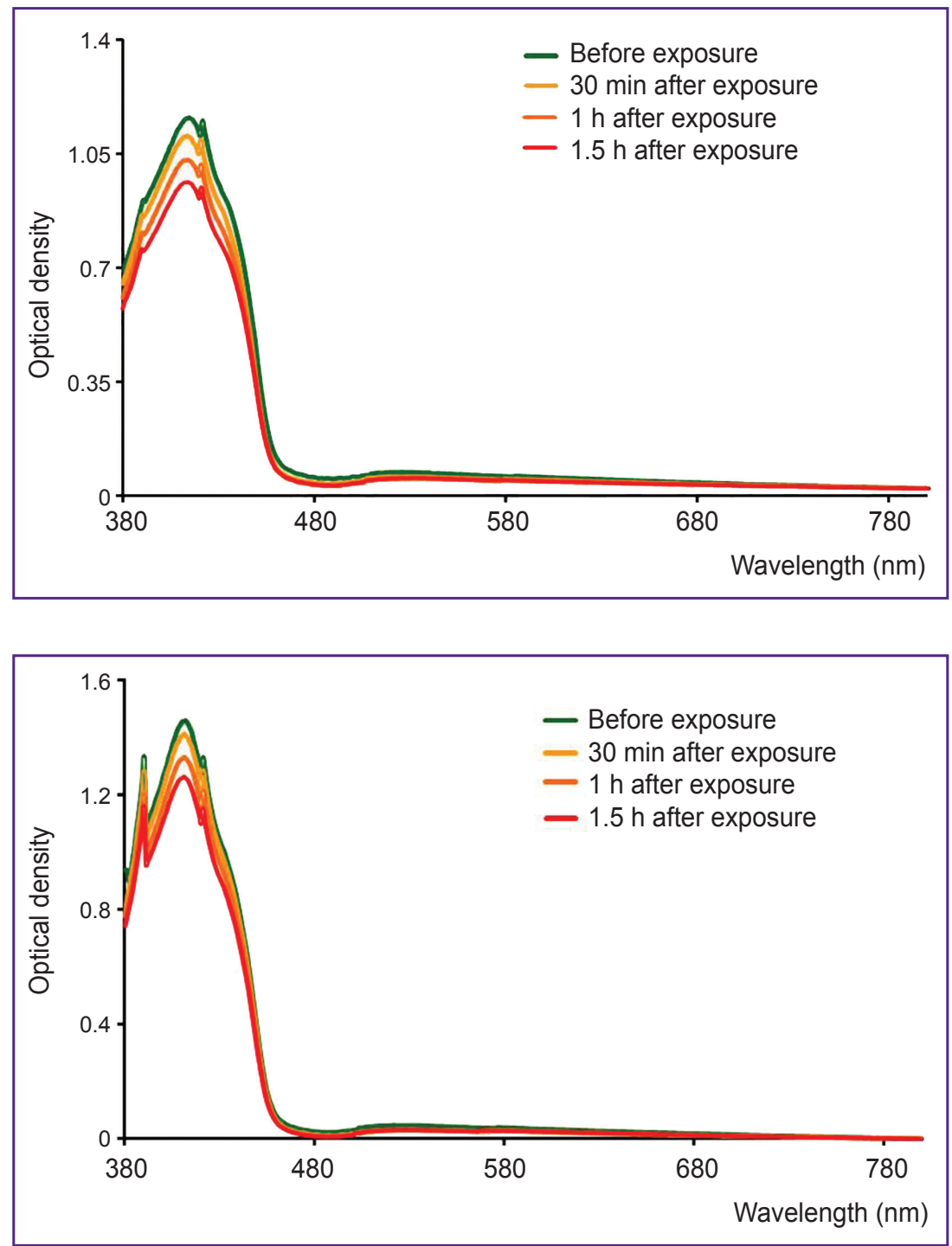

Efficiency of photodestruction of singlet oxygen trap under the action of two infrared lasers with different radiation modes

\begin{tabular}{ccc}
\hline $\begin{array}{c}\text { Medium } \\
\text { (human blood } \\
\text { plasma) }\end{array}$ & $\begin{array}{c}\text { The rate of DPIBF discoloration (standard units) } \\
\text { normalized to the laser radiation power }\end{array}$ \\
\cline { 2 - 3 } & $\begin{array}{c}\text { Nanosecond pulsed } \\
\text { laser radiation }\end{array}$ & $\begin{array}{c}\text { Continuous } \\
\text { laser radiation }\end{array}$ \\
\hline Specimen 1 & 0.042 & 0.003 \\
\hline Specimen 2 & 0.041 & 0.006 \\
\hline Specimen 3 & 0.038 & 0.004 \\
\hline
\end{tabular}

to the rate of the trap optical density decrease normalized to $1 \mathrm{~W}$ power over $30 \mathrm{~min}$ of laser radiation. Each number is an average value for the three independent measurements (statistical error $\pm 5 \%$ ).

It is seen from the table, that discoloration of DPIBF is actually 10 times greater in case of using nanosecond pulsed laser compared to the application of continuous mode laser. The result obtained can be explained by the fact that ultrashort high power pulses contribute to the time of oxygen relaxation in the tissues and probable summation of laser photon energy, and therefore filling the tissues with singlet oxygen.

Thus, since DPIBF molecules (of the chemical trap) have the absorption maximum only in UV- and visible region of the spectrum and do not absorb laser $\mathrm{IR}$ radiation, the effect is caused by the direct laser excitation of the dissolved oxygen, whose absorption band corresponds to the spectral region of laser radiation. Comparison of the effect of nanosecond pulsed laser and that of the laser generating continuous IR radiation shows that the efficiency of DPIBF discoloration is substantially greater when exposed to the pulsed laser compared to the laser with the continuous radiation with equal wavelength approaching to the maximum of oxygen absorption.

Conclusion. The results of evaluating the efficiency 
of singlet oxygen excitation in blood plasma in vitro speak of a great functional potential of the developed laser model generating nanosecond pulse radiation. Dimensions and the unique characteristics of the laser allow it to be applied in the form of a module in robotic units for maxillofacial surgery.

Study Funding and Conflicts of Interest. Subsidy 056-00139-16 was granted in the frame of the state task of the Ministry of Health of the Russian Federation. The authors have no conflict of interests to declare.

\section{References}

1. Lehnert M.W. Lasers in medicine and dentistry. Northwest Dent 1996; 75(1): 17-22.

2. Goonewardene S.S., Persad R. Robotic radical prostatectomy, day-case surgery and cardiac health: an opposing paradigm? J Robot Surg 2015, 9(4): 355, https://doi. org/10.1007/s11701-015-0537-9.

3. Propedevticheskaya stomatologiya [Propaedeutic dentistry]. Pod red. Bazikyana E.A. [Bazikyan E.A. (editor)]. Moscow: GEOTAR-Media, 2008; 768 p.

4. Borisenko G.G., Lukina G.I., Bazikyan E.A., Kovaleva A.M. Assessment of morphological changes in oral mucosa in patients with gastrointestinal pathology. Klinicheskaya meditsina 2009; 87(6): 36-38.

5. Krasnovsky A.A. Jr., Drozdova N.N., Roumbal Ya.V., Ivanov A.V., Ambartzumian R.V. Biophotonics of molecular oxygen: activation efficiencies upon direct and photosensitized excitation. Chin Opt Lett 2005; 3: S1-S4.

6. He G.S., Lin T.-C., Dai J., Prasad P.N., Kannan R., Dombroskie A.G., Vaia R.A., Tan L.-S. Degenerate two-photonabsorption spectral studies of highly two-photon active organic chromophores. J Chem Phys 2004; 120(11): 5275, https://doi. org/10.1063/1.1647537.

7. Chunikhin A.A., Bazikyan E.A., Krasnovsky A.A., Syrnikova N.V., Chobanyan A.G. The prospects for the improvement of the minimally invasive laser technologies for the photodynamic treatment of dental pathologies. Rossiiskaya stomatologiya 2015; 8(2): 70-74, https://doi.org/10.17116/ rosstomat20158271-74. 